\title{
Discrimination of spatial and temporal intervals defined by three light flashes: Effects of spacing on temporal judgments and of timing on spatial judgments
}

\author{
C. E. COLLYER \\ University of Rhode Island, Kingston, Rhode Island 02881
}

\begin{abstract}
Observers were presented stimulus patterns consisting of a sequence of three laterally displaced light flashes, which defined two spatial intervals and two temporal intervals. The position and time of the second flash were varied factorially, and observers were asked to make relative judgments of either the two spatial intervals or the two temporal intervals. "Induction" effects of stimulus timing on spatial judgments and of stimulus spacing on temporal judgments were both found; however, the directionality of these effects differed between subjects. The results are inconsistent with the hypothesis, derived from previous findings, that such effects are determined primarily by a tendency toward perceiving constant velocity of apparent motion; it is proposed that the directionality of the induction effects is determined largely by the strategy adopted by the observer for combining spatial and temporal stimulus information.
\end{abstract}

Three successive, laterally displaced flashes of light (or three successive, laterally displaced tactile pulses) define two successive temporal intervals and two adjoining spatial intervals. An observer may be asked to judge the relative magnitude of the two times, or of the two distances. Figure 1 illustrates the spacing and timing of three successive stimuli arrayed along one spatial dimension, and introduces some terms that will be useful in describing particular stimulus patterns. Part a of the figure shows the spatial configuration of the three stimulus positions. Part $b$ shows the time course of their presentation. Part $c$ is a "space-time" diagram which graphically summarizes the spatial and temporal parameters of the stimulus pattern. (The dashed lines on the diagram represent hypothetical patterns of apparent motion, which will be referred to later.)

The present study employs visual stimuli and is concerned with the way in which the relative magnitudes of $s_{1}$ and $s_{2}$ influence an observer's judgment of the relative magnitude of $t_{1}$ and $t_{2}$, and vice versa.

Helson (1930; Helson \& King, 1931) investigated spatial judgments in the cutaneous modality. It was demonstrated that an observer's spatial judgment of the relative magnitudes of $s_{1}$ and $s_{2}$ is strongly influenced by the relative magnitudes of $t_{1}$ and $t_{2}$. In general, if $t_{1}$ exceeded $t_{2}$, then $s_{1}$ was judged greater than $s_{2}$. This tendency in spatial judgments was termed the tau effect by Helson; this term is also used to refer to the visual analogue of the effect, which was reported by Geldreich (1934).

It has also been found (Abe, 1935; Cohen et al., 1953, 1955; Price-Williams, 1954) that an observer's temporal judgment of the relative magnitudes of $t_{1}$ and $t_{2}$ is strongly influenced by the relative magnitudes of $s_{1}$ and $s_{2}$. If $s_{1}$ exceeded $s_{2}$, then $t_{1}$ was judged

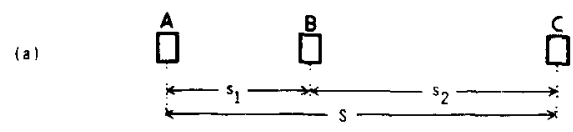

(b)
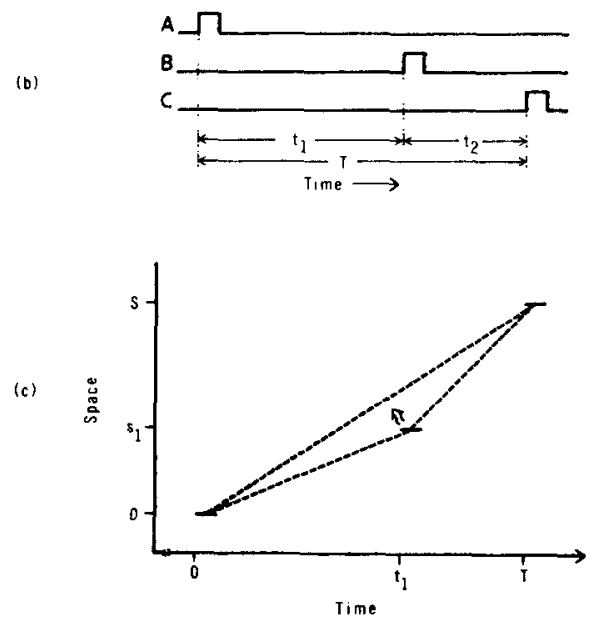

Figure 1. Parameters of a dynamic stimulus pattern consisting of three successive, laterally displaced stimuli. (a) Spatial configuration of stimulus positions. (b) Time course of stimulus presentation. (c) "Space-time diagram" incorporating both spatial and temporal parameters. 
to be greater than $t_{2}$. This tendency in temporal judgments was termed the kappa effect by Cohen et al. (1953).

The effect of timing on spatial judgments seems to be a distortion in the apparent relative magnitudes of $s_{1}$ and $s_{2}$, making them more similar to the relative magnitudes of $t_{1}$ and $t_{2}$. Conversely, the effect of spacing on temporal judgments seems to be a temporal distortion in the direction of the relative magnitudes of $s_{1}$ and $s_{2}$. Both effects seem consistent with the hypothesis that observers have a perceptual tendency toward equalizing the ratios $t_{1} / t_{2}$ and $s_{1} / s_{2}$. This hypothesis is formally equivalent to the hypothesis that observers tend to equalize the ratios $s_{1} / t_{1}$ and $s_{2} / t_{2}$, which define velocities. (It seems reasonable that the corresponding apparent velocities are monotonic increasing functions of these ratios.) A graphic representation of this hypothesis is given in Figure 1c. The arrow represents a hypothetical perceptual tendency to distort the spatial and temporal coordinates of, in this example, the second stimulus, in a way that decreases the difference between $s_{1} / t_{1}$ and $s_{2} / t_{2}$. A version of this "constant velocity hypothesis" is implied by Cohen et al. (1955) in their discussion of the kappa effect; in a recent paper (Collyer, 1976), ${ }^{1}$ it was shown that such a hypothesis is consistent with the directionality of four dynamic perceptual phenomena, including the tau and kappa effects. One other of these phenomena, the induced asynchrony effect, will be briefly described.

A dynamic stimulus pattern consisting of four lights arrayed horizontally is represented in Figure 2. Two initially illuminated lights, $\mathbf{A}$ and $\mathbf{B}$, are extinguished at different times, followed after a brief dark interval by the simultaneous onsets of lights $\mathrm{C}$ and D. C and D are positioned at distances $s_{1}$ and $s_{2}$, respectively, from $A$ and $B$, and, in this simple case, $s_{1}=s_{2}$. In the diagram, B is extinguished before A; under these conditions, an observer would tend to judge the onset at $D$ to occur before the onset at $C$, as indicated by the arrow pointing leftward. This tendency is termed the induced asynchrony effect (IAE). In general, the onset of that second stimulus (C or D), which is closer in space to the initially extinguished stimulus (A or B), will tend to be judged earlier.

The dashed lines in Figure 2 represent hypothetical patterns of apparent motion between $A$ and $C$ and between $B$ and $D$. The velocities of these apparent motions, if this aspect of perception were veridical, would be given by $v_{1}=s_{1} / t_{1}$ and $v_{2}=s_{2} / t_{2}$, respectively, with $v_{1}>v_{2}$ because $t_{1}<t_{2}$. However, the existence of the IAE suggests that, phenomenally, $t_{2}$ is decreased relative to $t_{1}$ by the apparent precedence of the onset of stimulus $D$. Hence, the IAE might be construed as a tendency to distort $\mathrm{v}_{2}$ in the

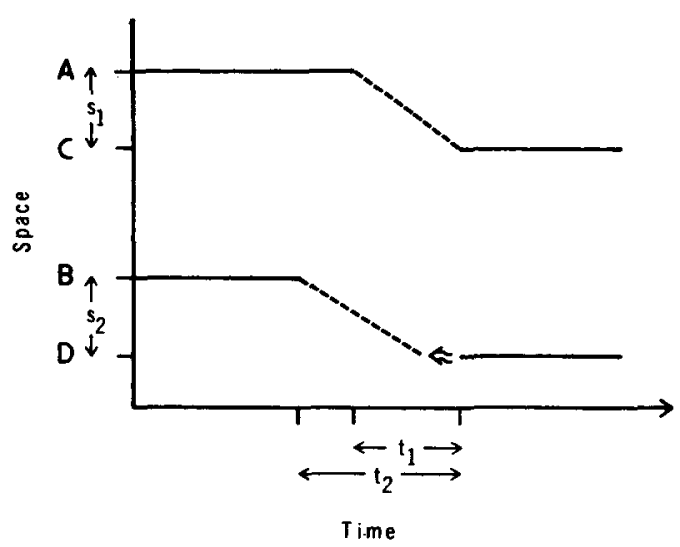

Figure 2. Space-time diagram representing the induced asynchrony effect. The arrow signifies an observer's tendency to judge the onset of Stimulus $D$ to precede the onset of $\mathbf{C}$ if the offset of Stimulus B precedes the off fet of $A$.

direction of equality with $\mathrm{v}_{1}$. It is in this sense that the IAE, like the tau and kappa effects, appears consistent with a "constant-velocity hypothesis."

It is intriguing that several phenomena should display properties which appear consistent with a general hypothesis. However, there are two important considerations which prevent us from making a strong case for the unity of these phenomena. First, as Figure 1c suggests, distortions of both space and time should occur when both the timing and the spacing of three stimuli are "uneven." However, it has not yet been shown that both distortions, tau and kappa, can be obtained using the same set of stimulus patterns and varying only the observer's task (temporal or spatial judgment). Such a demonstration would require relatively small, confusable differences between both $s_{1}$ and $s_{2}$ and between $t_{1}$ and $t_{2}$, because, while each difference would, in one task, be a value of the "inducing" variable, in the other task it would be the quantity being judged. In order to obtain robust distortions, however, previous investigators have employed large differences on the "inducing" dimension in each task. The constantvelocity hypothesis requires that qualitatively similar, if attenuated, distortions should be observed when the inducing differences are small.

A second important consideration is the difference among previous studies in the duration of the interval within which the manipulated stimulus events occur. Much of Cohen's experimental work on the kappa effect (e.g., Cohen, 1967; Cohen et al., 1953) has been done with $T=1.5 \mathrm{sec}$ (see Figure 1). Higher values have also been used. Helson and King (1931) employed values of $T$ ranging from 600 to $900 \mathrm{msec}$. In the experiments on the IAE (Collyer, 1976), however, the maximum time interval between either offset and either onset was only $150 \mathrm{msec}$; this 
was designed to eliminate the possibility of multiple eye fixations, which exists in the previous studies. Thus, in different studies, the time interval within which the information-carrying events in a stimulus pattern occur varies considerably. To evaluate the generality of the constant-velocity hypothesis, it seems desirable to collect data involving both temporal and spatial judgments while holding the value of $T$ constant; furthermore, it is appropriate to keep the value of $T$ small enough to allow the observer only one fixation.

\section{EXPERIMENT 1}

This experiment employed stimulus patterns consisting of three laterally displaced lights which were illuminated briefly and in succession from left to right on each trial (as in Figure 1). The timing and positions of the peripheral lights never varied; however, the center light could be presented in one of three positions, and at one of three times, on each trial. Thus there was a total of nine stimulus patterns. The object of the experiment was to vary the question asked of the subject-sometimes he judged the relative timing of the center light, and sometimes its relative spacing-and to discover how the judgments in each case were influenced by the time and position of the center light.

\section{Method}

Subjects. Three males, aged 17-20, served as paid observers. They had answered an advertisement in a local newspaper.

Apparatus and Stimuli. Stimuli consisted of $6 \times 4$ matrices of closely spaced points on a Tektronix 602 CRT screen, and had the appearance of illuminated rectangular patches. A train of three laterally separated stimuli constituted a stimulus pattern. A DEC PDP-12 computer controlled the spacing and timing of stimuli within stimulus patterns, as well as the timing of all trial intervals and the recording of responses. The sequence of events within each trial was as follows. A fixation field, consisting of a horizontal row of 200 illuminated points and subtending about $4^{\circ}$ visual angle, was presented for $100 \mathrm{msec}$, followed by a 500 -msec dark interval. Three light flashes were then presented, as in Figure 1 , with $S=s_{1}+s_{2} \cong 4^{\circ}, T=t_{1}+t_{2}=160 \mathrm{msec}$, and the duration of each flash equal to $10 \mathrm{msec}$. The flashes were located about $1^{\circ}$ below the fixation line. The programmed duration of the response interval was $2,000 \mathrm{msec}$; observers learned during practice to respond within this interval. Viewing distance was about $30 \mathrm{in}$. Note that the values of $t_{1}$ and $s_{1}$ are sufficient to specify a particular stimulus pattern.

Design and Procedure. Three values of $s_{1}$ and three values of $t_{1}$ were combined factorially for each observer; on each trial, the stimulus pattern presented represented one of the nine possible combinations of $s_{1}$ and $t_{1}$ values. This aspect of the design is illustrated in Figure 3.

The exact values of $s_{1}$ and $t_{1}$ for each observer are given in Table 1. Different ranges of $t_{1}$ were chosen for each observer on the basis of preliminary data, in order to obtain roughly equal error rates.

The particular combination of $s_{1}$ and $t_{1}$ employed on a given trial was randomly determined by sampling with replacement from the set of nine such combinations; the a priori probability of sampling each combination was 1/9.

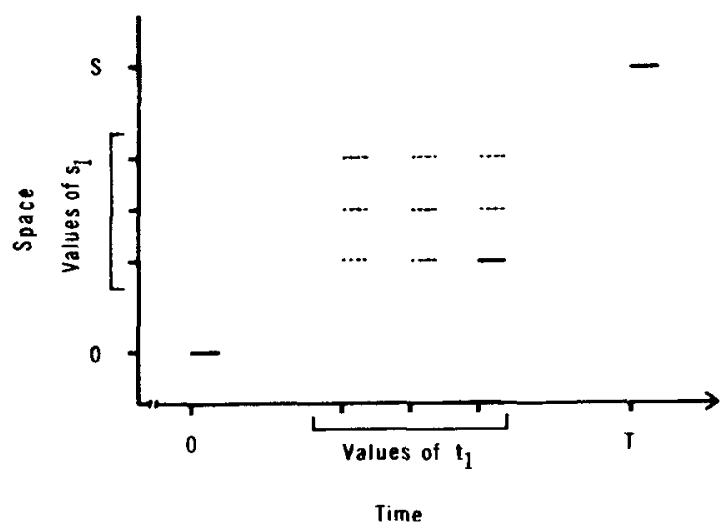

Figure 3. Space-time diagram illustrating the way in which values of $t_{1}$ and of $s_{1}$ were factorially combined to produce nine different stimulus patterns. One of the nine combinations was sampled on each trial.

There were two experimental conditions, which differed only in the judgment the observer was instructed to make. In the temporal condition, the observer's task was to report whether the second temporal interval appeared longer (an $R_{1}$ response) or shorter (an $R_{2}$ response) in duration than the first. In the spatial condition, the task was to report whether the second spatial interval appeared longer (an $R_{1}$ response) or shorter (an $R_{2}$ response) in extent than the first.

Observers were seated in an acoustical chamber, and viewed the CRT display through a tunnel external to the window of the chamber. They were instructed to fixate "about in the middle" of the fixation line when it appeared. A fixed central fixation stimulus was avoided because it could easily serve as a reference stimulus for spatial judgments. The experimenter further instructed each observer to make spatial or temporal judgments at the beginning of each session. In both conditions, responses were made by pressing a button on the left $\left(R_{1}\right)$ or on the right $\left(R_{2}\right)$ of a response panel.

Daily sessions consisted of four blocks of 100 trials, separated by short rest periods. Observers L.R., M.C., and C.N. received 13,7 , and 10 sessions, respectively, of preliminary experience with the experiment. Observers L.R. and M.C. were each run for four data sessions under each condition; observer C.N. was run for five data sessions under each condition. The data of main interest consisted of the proportions of $\mathbf{R}_{2}$ responses given each

Table 1

Values of $s_{1}$ in Degrees Visual Angle, and of $t_{1}$ in Milliseconds for Each Condition and Observer, in Experiments 1 and 2

\begin{tabular}{|c|c|c|c|}
\hline Condition & Observer & Values of $s_{1}$ & Values of $t_{1}$ \\
\hline \multicolumn{4}{|c|}{ Experiment 1} \\
\hline Both & & & \\
\hline Conditions & $\begin{array}{l}\text { L.R. } \\
\text { M.C. } \\
\text { C.N. }\end{array}$ & $\begin{array}{l}1.94,2.00,2.06 \\
1.94,2.00,2.06 \\
1.94,2.00,2.06\end{array}$ & $\begin{array}{l}56,80,104 \\
50,80,110 \\
38.80,122\end{array}$ \\
\hline \multicolumn{4}{|c|}{ Experiment 2} \\
\hline Temporal & & & \\
\hline Judgments & $\begin{array}{l}\text { L.R. } \\
\text { M.C. } \\
\text { C.N. }\end{array}$ & $\begin{array}{l}1.75,2.00,2.25 \\
1.75,2.00,2.25 \\
1.75,2.00,2.25\end{array}$ & $\begin{array}{l}56,80,104 \\
50,80,110 \\
38,80,122\end{array}$ \\
\hline $\begin{array}{l}\text { Spatial } \\
\text { Judgments }\end{array}$ & $\begin{array}{l}\text { L.R. } \\
\text { M.C. } \\
\text { C.N. }\end{array}$ & $\begin{array}{l}1.94,2.00,2.06 \\
1.94,2.00,2.06 \\
1.94,2.00,2.06\end{array}$ & $\begin{array}{l}20,80,140 \\
20,80,140 \\
20,80,140\end{array}$ \\
\hline
\end{tabular}


combination of $s_{1}$ and $t_{1}$ values, for each condition and observer. The number of observations on which each such proportion is based varies, but in no case is less than 145 .

Sessions in Experiment 1 were interleaved with sessions in Experiment 2, in order to minimize differences in amount of practice between the two experiments. This was done in such a way that (1) observers performed temporal and spatial judgments on alternate days; and (2) a replication of both experiments (i.e., two sessions in Experiment 1 and two sessions in Experiment 2) was completed every 4 days.

\section{Results}

Preliminary statistical analysis. It is of interest initially to determine whether the independent variables, $s_{1}$ and $t_{1}$, affected the observers' judgments in each condition. For each of the six sets of data (three observers, two conditions each), the raw response frequencies conditional on each combination of $s_{1}$ and $t_{1}$ values were analyzed by a method described by Goodman (1969) for partitioning chi square in factorial experiments yielding frequency data. The results of this analysis are summarized in Table 2.

The judged variable clearly influenced all three observers' judgments, in both conditions. In four of the six sets of data, the nonjudged variable had a statistically significant effect (three at $p<.001$ and one at $p<.05)$. There was no significant interaction between $s_{1}$ and $t_{1}$ in any of the six cases.

Temporal condition. The observed proportions, $P_{T}\left(R_{2} \mid s_{1}, t_{1}\right)$ of $R_{2}$ responses are entered in Table 3 for each stimulus pattern and observer.

These proportions are plotted in Figure 4 as a function of $s_{1}$ for each value of $t_{1}$. (The ordinate in Figures 4 through 7 is a Gaussian transformation, or probit, scale. A model which leads to this scaling will be suggested in the Discussion.)
Table 2

Levels of Significance Attained by a Chi-Square Statistic for Each Source of Variation in Each Observer's Response Frequency Data, in Experiment 1

\begin{tabular}{lcccc}
\hline Condition & Observer & \multicolumn{3}{c}{ Source of Variation } \\
\hline \multirow{3}{*}{ Temporal } & & $\mathrm{s}_{1}$ & $\mathrm{t}_{\mathbf{1}}$ & Interaction \\
\cline { 3 - 5 } & L.R. & .11 & $*$ & .61 \\
& M.C. & .46 & $*$ & .89 \\
Spatial & C.N. & $*$ & $*$ & .95 \\
& L.R. & $*$ & .05 & .23 \\
& M.C. & $*$ & $*$ & .76 \\
& C.N. & $*$ & $*$ & .11 \\
\hline
\end{tabular}

${ }^{*}$ Denotes $p<.001$.

Table 3

Proportions of $\mathbf{R}_{2}$ Responses Given Condition, Observer, and Stimulus Pattern, Experiment 1

Temporal Judgment Condition

Observer

\begin{tabular}{|c|c|c|c|c|c|c|c|c|c|}
\hline \multirow[t]{2}{*}{$s_{1}$} & \multicolumn{3}{|c|}{$\begin{array}{c}\text { L.R. } \\
t_{1}\end{array}$} & \multicolumn{3}{|c|}{$\begin{array}{c}\text { M.C. } \\
t_{1}\end{array}$} & \multicolumn{3}{|c|}{$\begin{array}{c}\text { C.N. } \\
t_{1}\end{array}$} \\
\hline & 56 & 80 & 104 & 50 & 80 & 110 & 38 & 80 & 122 \\
\hline $\begin{array}{l}2.06 \\
2.00 \\
1.94\end{array}$ & $\begin{array}{l}.10 \\
.14 \\
.22\end{array}$ & $\begin{array}{l}.46 \\
.55 \\
.64\end{array}$ & $\begin{array}{l}.92 \\
.94 \\
.93\end{array}$ & $\begin{array}{l}.23 \\
.24 \\
.29\end{array}$ & $\begin{array}{l}.43 \\
.48 \\
.48\end{array}$ & $\begin{array}{l}.95 \\
.95 \\
.97\end{array}$ & $\begin{array}{l}.19 \\
.13 \\
.09\end{array}$ & $\begin{array}{l}.59 \\
.52 \\
.41\end{array}$ & $\begin{array}{l}.96 \\
.94 \\
.93\end{array}$ \\
\hline & \multicolumn{9}{|c|}{ Spatial Judgment Condition } \\
\hline 1 & \multicolumn{3}{|c|}{$\begin{array}{c}\text { L.R. } \\
t_{1}\end{array}$} & \multicolumn{3}{|c|}{$\begin{array}{c}\text { M.C. } \\
t_{1}\end{array}$} & \multicolumn{3}{|c|}{$\begin{array}{c}\text { C.N. } \\
t_{1}\end{array}$} \\
\hline & 56 & 80 & 104 & 50 & 80 & 110 & 38 & 80 & 122 \\
\hline $\begin{array}{l}2.06 \\
2.00 \\
1.94\end{array}$ & $\begin{array}{l}.82 \\
.56 \\
.19\end{array}$ & $\begin{array}{l}.75 \\
.43 \\
.19\end{array}$ & $\begin{array}{l}.72 \\
.49 \\
.22\end{array}$ & $\begin{array}{l}.83 \\
.65 \\
.50\end{array}$ & $\begin{array}{l}.69 \\
.54 \\
.40\end{array}$ & $\begin{array}{l}.54 \\
.33 \\
.24\end{array}$ & $\begin{array}{l}.94 \\
.79 \\
.52\end{array}$ & $\begin{array}{l}.91 \\
.69 \\
.33\end{array}$ & $\begin{array}{l}.60 \\
.17 \\
.03\end{array}$ \\
\hline
\end{tabular}
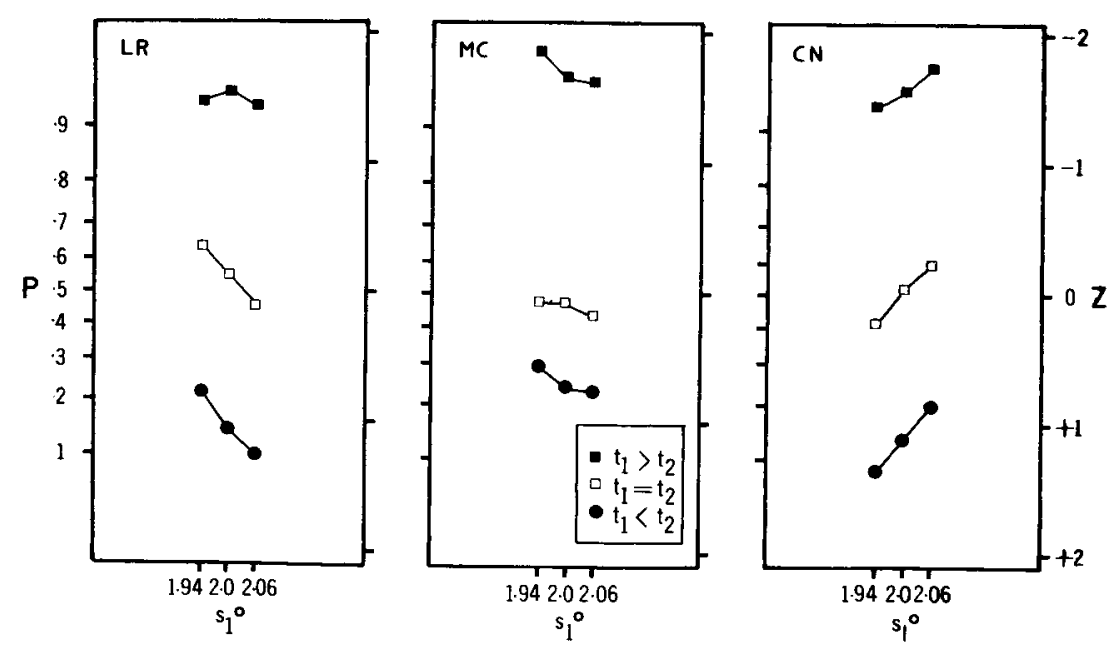

Figure 4. Experiment 1, temporal condition. The observed proportion of $\mathbf{R}_{2}$ responses is plotted against $s_{1}$. Positive slopes would indicate a kappa-like effect. 
It is clear from the vertical separation and the ordering of the curves in Figure 4 that all three observers were able to perform the temporal discrimination. The only subject to show a statistically significant effect of $s_{1}$, observer C.N., displays the positive slope indicative of a kappa-like effect, and consistent with a constant-velocity hypothesis. There is some suggestion of a systematic effect of $s_{1}$, but opposite in direction to the kappa pattern, for L.R. and M.C.; however, more data would be required to further assess this possibility.

Spatial condition. The observed proportions, $P_{S}\left(R_{2} \mid s_{1}, t_{1}\right)$, of $R_{2}$ responses are entered in Table 3 . These data are plotted in Figure 5 as a function of $t_{1}$ for each value of $s_{1}$

In Figure 5, the vertical separation and ordering of the curves associated with each value of $s_{1}$ indicate the observers' ability to perform the spatial discrimination. The effect of temporal variation on spatial judgments is shown by the slopes of the curves. None of the observers' curves display the positive slope that would indicate the presence of a tau-like effect, and support a constant velocity hypothesis. The slopes for observers M.C. and C.N. are clearly negative, while those for observer L.R. do not seem to show a monotonic relationship between $P\left(R_{2}\right)$ and $t_{1}$.

The data indicate that, in general, space and time are not judged independently; however, only one observer displayed a kappa-like effect in the temporal condition, and none of the three observers displayed a tau-like effect in the spatial condition. The presence of statistically significant "inducing" effects associated with reversals of the tau pattern in the spatial condition provides evidence against the primacy of any perceptual tendency toward constant velocity, at least in this experiment.

An alternative to the constant-velocity hypothesis is that observers adopt individual strategies for combining spatial and temporal information. That is, the effects of the inducing dimension in each condition may reflect the observer's selection of "software" for processing information rather than simple sensory or "hard-wired" tendencies.

\section{EXPERIMENT 2}

In Experiment 1, relatively small ranges of values were employed on the "inducing" dimension in each condition, in order that the same set of nine stimulus patterns could be judged in each condition. It is possible that, as a result of this feature of the experiment, the "level of induction" was too low to produce consistent tau and kappa effects. The difference between Experiments 1 and 2 was in the range of variation in the nonjudged, "inducing" dimension in each task; like previous experiments on the tau and kappa effects, Experiment 2 employed a relatively wide range of $s_{1}$ values in the temporal condition and of $t_{1}$ values in the spatial condition. It is of interest to examine the inducing effects under these "higher levels" of spatial and temporal induction.

\section{Method}

The subjects, apparatus, and procedure of Experiment 2 were identical to those of Experiment 1. The values of $s_{1}$ in the spatial condition and of $t_{1}$ in the temporal condition were also the same as before. However, the values of $s_{1}$ in the temporal condition and of $t_{1}$ in the spatial condition were varied over larger ranges than in Experiment 1. The exact values of both variables are given in Table 1 for each condition and observer. The values
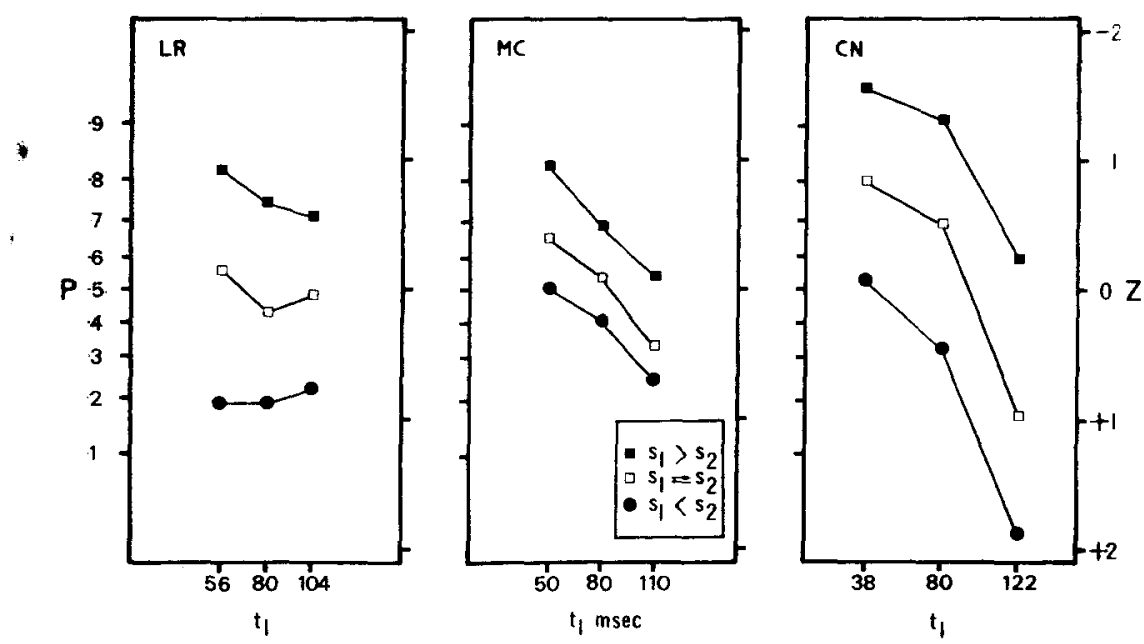

Figure 5. Experiment 1, spatial condition. The observed proportion of $R_{2}$ responses is plotted against $t_{1}$. Positive slopes would indicate a tau-like effect. 
of the nonjudged variable in each condition were selected to span about the smallest range of variation which gave virtually errorfree discrimination when judged in the preliminary practice sessions. Observer L.R. was run for four data sessions under each condition; observers M.C. and C.N. were run for five sessions under each condition.

\section{Results}

Preliminary statistical analysis. Goodman's (1969) technique for partitioning chi square was applied to the raw response frequencies, as in Experiment 1. The results of this analysis are summarized in Table 4.

Again, the effect of the judged variable in each condition was highly significant $(p<.001)$. The nonjudged variable had a statistically significant effect in five of the six cases (four at $p<.001$ and one at $p<.05)$. This confirms the conclusion that, in general, these judgments are not independent of the inducing variable. The interaction term in this analysis attains statistical significance in two of the six sets of data (both at $\mathrm{p}<.05$ ).

Temporal condition. The observed proportions, $\mathbf{P}_{\mathrm{T}}\left(\mathbf{R}_{\mathbf{2}} \mid \mathrm{s}_{1}, \mathrm{t}_{1}\right)$, are entered in Table 5 for each condition and observer.

These data are plotted in Figure 6 as a function of $s_{1}$ for each value of $t_{1}$. The abscissa is drawn to the same scale as in Figure 4 to facilitate comparison between Experiments 1 and 2 .

Increasing the range of $s_{1}$ in Experiment 2 did not result in a consistent pattern of kappa-like positive slopes. From a comparison of Figures 4 and 6 , it appears that roughly the same pattern of inducing effects resulted in this experiment as in Experiment 1 , for temporal judgments. The curves for observer C.N. again show positive slopes; those for
Table 4

Levels of Significance Attained by a Chi-Square Statistic for Each Source of Variation in Each Observer's Response Frequency Data, in Experiment 2

\begin{tabular}{lcccc}
\hline Condition & Observer & \multicolumn{3}{c}{ Source of Variation } \\
\hline \multirow{3}{*}{ Temporal } & & $\mathrm{s}_{1}$ & $\mathrm{t}_{1}$ & Interaction \\
\cline { 2 - 5 } & L.R. & $*$ & $*$ & .02 \\
\multirow{3}{*}{ Spatial } & M.C. & .02 & $*$ & .59 \\
& C.N. & $*$ & $*$ & .01 \\
& L.R. & $*$ & .44 & .07 \\
& M.C. & $*$ & $*$ & .32 \\
& C.N. & $*$ & $*$ & .50 \\
\hline
\end{tabular}

*Denotes $p<.001$.

L.R. show generally negative slopes; and those for M.C. are quite shallow.

Spatial condition. The observed proportions, $P_{S}\left(R_{2} \mid s_{1}, t_{1}\right)$, of $R_{2}$ responses are entered in Table 5 and plotted in Figure 7 as a function of $t_{1}$ for each value of $s_{1}$. The abscissa is drawn to the same scale as in Figure 5.

Increasing the range of $t_{1}$ did not produce consistent positive slopes in Figure 7. Comparison of Figures 5 and 7 seems to show that a similar pattern of inducing effects was obtained in the spatial conditions of the two experiments. The curves for observer L.R. display little or no slope, while those of observers M.C. and C.N. show negative slopes.

Of the 12 sets of data in the two experiments, nine displayed statistically significant effects of the nonjudged variable. Of these, only two seem clearly consistent with the constant-velocity hypothesis (observer C.N.'s temporal judgments in both experiments), while several cases seem to contradict it. It is reasonable to conclude that the directionality of
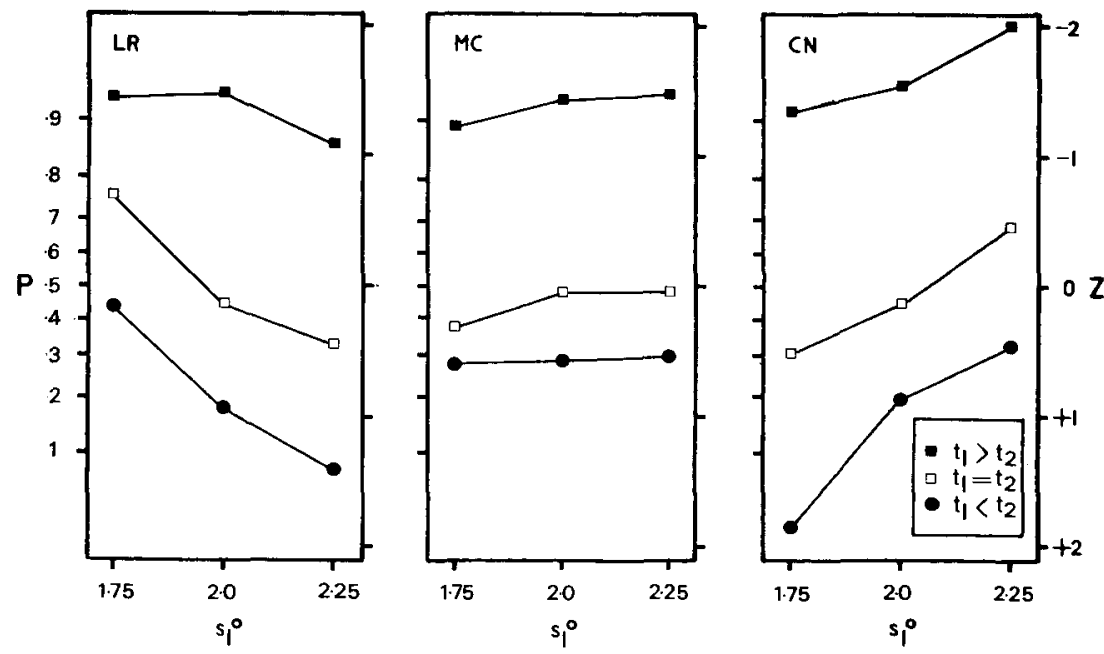

Figure 6. Experiment 2, temporal condition. Compare with Figure 4, noting that the range of $s_{1}$ differs between the two experiments. 

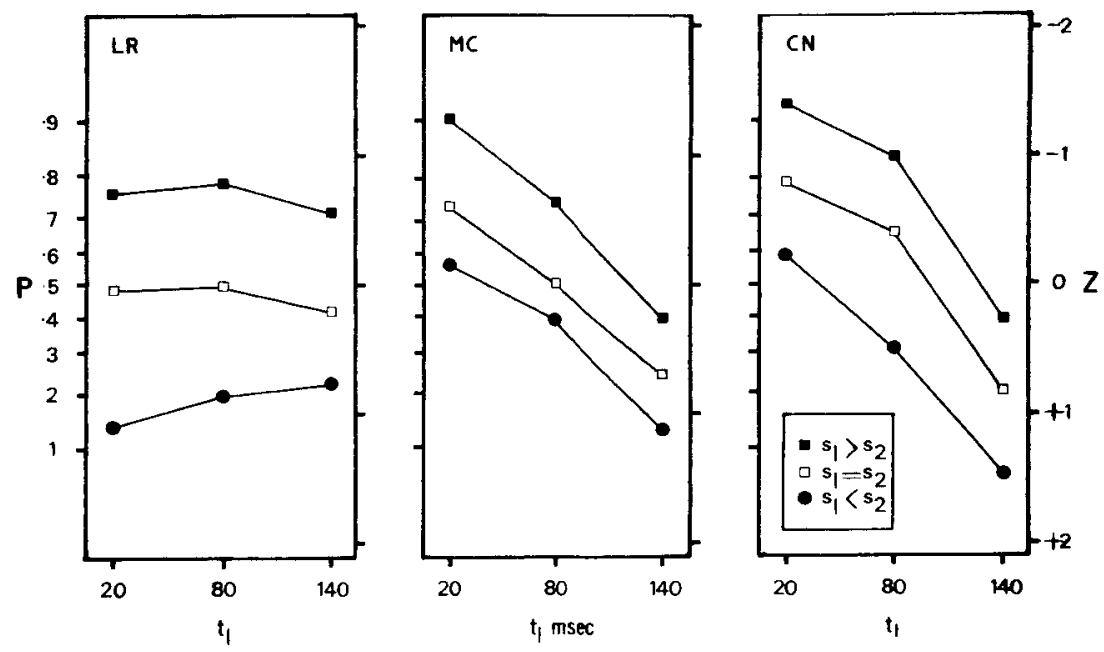

Figure 7. Experiment 2, spatial condition. Compare with Figure 5, noting that the range of $t_{1}$ differs between the two experiments.

such inducing effects is not predetermined by an inherent perceptual tendency.

\section{DISCUSSION}

In general, spatial judgments are not independent of temporal relations within stimulus patterns, and temporal judgments are not independent of spatial relations. Individual differences seem to exist between observers in the pattern of these departures from independence. The disparity between the present results and those of earlier experiments on the tau and kappa effects, suggests (1) that the original effects have previously unsuspected boundary conditions, which require investigation; and (2) that future theoretical development in the area of relative spatial and temporal judgments must include models

Table 5

Proportions of $\mathbf{R}_{2}$ Responses Given Condition, Observer, and Stimulus Pattern, Experiment 2

Temporal Judgment Condition

Observer

\begin{tabular}{|c|c|c|c|c|c|c|c|c|c|}
\hline \multirow[t]{2}{*}{$\mathrm{s}_{1}$} & \multicolumn{3}{|c|}{$\underset{t_{1}}{\text { L.R. }}$} & \multicolumn{3}{|c|}{$\underset{t_{1}}{M . C .}$} & \multicolumn{3}{|c|}{$\begin{array}{c}\text { C.N. } \\
t_{1}\end{array}$} \\
\hline & 56 & 80 & 104 & 50 & 80 & 110 & 38 & 80 & 122 \\
\hline 2.25 & .08 & .33 & .86 & .30 & .49 & .93 & .33 & .68 & .98 \\
\hline 2.00 & .18 & .45 & .93 & .28 & .48 & .93 & .20 & .45 & .94 \\
\hline 1.75 & .44 & .76 & .93 & .27 & .38 & .89 & .03 & .31 & .92 \\
\hline \multicolumn{10}{|c|}{ Spatial Judgment Condition } \\
\hline & \multicolumn{9}{|c|}{ Observer } \\
\hline$s_{1}$ & \multicolumn{3}{|c|}{$\begin{array}{c}\text { L.R. } \\
t_{1}\end{array}$} & \multicolumn{3}{|c|}{$\begin{array}{c}\text { M.C. } \\
t_{1}\end{array}$} & \multicolumn{3}{|c|}{$\begin{array}{c}\text { C.N. } \\
t_{1}\end{array}$} \\
\hline & 20 & 80 & 140 & 20 & 80 & 140 & 20 & 80 & 140 \\
\hline .06 & .76 & .79 & .72 & .90 & .74 & .39 & .92 & .84 & .39 \\
\hline 00 & .49 & .50 & .41 & .73 & .51 & .24 & .79 & .66 & .20 \\
\hline 104 & .14 & .20 & .22 & .57 & .40 & .13 & .59 & .31 & .07 \\
\hline
\end{tabular}

which allow for a flexible, strategy-based integration of space and time information.

Why were consistent tau and kappa effects not recovered in these experiments? One possibility is that there were crucial procedural differences between the present study (which employed forcedchoice responses and randomized trial sequences) and earlier studies (which employed a variety of psychophysical procedures). At this point, it is not obvious which differences might be crucial. Another possibility is that the shorter duration of the stimulus patterns in the present study brought a different set of perceptual mechanisms into play. The latter alternative seems initially more promising to the author; further experiments will be aimed at determining whether the boundary conditions on tau and kappa are primarily temporal.

What do the present results suggest in regard to the apparent similarity between the IAE, on the one hand, and the tau and kappa effects, on the other? In Experiments 1 and 2, stimulus patterns associated with the previously reported tau and kappa effects were, in a sense, made more similar to the stimulus pattern used to evoke the IAE by greatly reducing their total duration. In these experiments, the tau and kappa effects were not found, which disconfirms the constant-velocity hypothesis for judgments of these patterns. These results do not allow us to flatly reject this hypothesis as an explanation of the IAE; however, they do suggest that any tendency toward perceiving constant velocity of apparent motion is not so predominant as to be reflected in all situations where it could be expected to operate. If such a tendency is not a primary determinant of judgments in the present study, then perhaps it isn't the important factor in the IAE either. However, a firm conclusion regarding the IAE cannot be based on the indirect evidence provided by the present study. 
One way to model the spatial and temporal judgments in the present experiments is to treat the observer's task as a statistical decision based on an internal code that (1) combines spatial and temporal stimulus information, and (2) is statistically "noisy." One such code might be denoted

$$
\mathbf{Y}=\mathbf{W}_{\mathbf{S}} \mathbf{S}^{*}+\mathbf{W}_{\mathbf{T}} \mathbf{T}^{*},
$$

where $S^{*}$ is a random variable whose expected value is a function of the relative magnitudes of $s_{1}$ and $S_{2} ; T^{*}$ is another random variable, independent of $S^{*}$, whose expected value is a function of the relative magnitudes of $t_{1}$ and $t_{2}$; and $W_{S}$ and $W_{T}$ are "weights" associated with $S^{*}$ and $T^{*}$, respectively. The code $Y$ would assume some value on each trial, and be compared with a criterion to effect response selection. The properties of this additive-effects model would be similar to those of both other statistical decision models (Green \& Swets, 1966) and other models for the algebraic combination of sources of stimulus information (Anderson, 1974). These properties are also characteristic of a recent model for letter detection (Kinchla, 1974; Kinchla \& Collyer, 1974).

We can say something about the correspondence between such a model and the data, with reference to Figures 4 through 7. The Gaussian transformation of response probability in the ordinate of each figure is appropriate if $\mathrm{Y}$ is assumed to be Gaussian. With respect to this transformation, the curves in each figure are predicted to be linear if $\mathbf{E}\left(S^{*}\right)$ is a linear function of $s_{1}$ in the temporal conditions and $E\left(T^{*}\right)$ is a linear function of $t_{1}$ in the spatial conditions. Furthermore, the curves in each figure are predicted to be equally spaced vertically if $E\left(S^{*}\right)$ is a linear function of $s_{1}$ in the spatial conditions and $E\left(T^{*}\right)$ is a linear function of $t_{1}$ in the temporal conditions. If these functions each have constant, positive slope, then the slopes of the data curves are theoretically determined by $W_{S}$ in the temporal conditions and $\mathbf{W}_{\mathrm{T}}$ in the spatial conditions, together with the variance of $Y$. Negative slopes imply negative weights, representing those cases where reversals of the tau and kappa effects were obtained.

In general, the predictions of the model, even with these severe simplifying assumptions, seem to capture many features of the data. A model of this sort may be worthy of further development in research aimed at discovering the conditions under which different rules apply to the way observers combine spatial and temporal aspects of dynamic stimulus displays.

\section{REFERENCES}

ABE, S. Experimental study on the co-relation between space and time. Tohoku Psychologica Folia, 1935, 3, 53-68.

Anderson, N. H. Algebraic models in perception. In E. C. Carterette \& M. P. Friedman (Eds.), Handbook of perception (Vol. II). New York: Academic Press, 1974.

COHEN, J. Psychological time in health and disease. Springfield, Ill: Thomas, 1967.

Cohen, J., Hansel, C. E. M., \& Sylvester, J. D. A new phenomenon in time judgment. Nature, 1953, 172, 901.

Cohen, J., Hansel, C. E. M., \& Sylvester, J. D. Interdependence in judgments of space, time and movement. Acta Psychologia, 1955, 11, 360-372.

COLLYER, C. E. The induced asynchrony effect: Its role in visual judgments of temporal order and its relation to other dynamic perceptual phenomena. Perception \& Psychophysics, 1976, 19. 47-54.

GeLDREICH, E. W. A lecture room demonstration of the visual tau effect. American Journal of Psychology, 1934, 46, 483.

Goodman, L. A. On partitioning chi-square and detecting partial association in three-way contingency tables. Journal of the Royal Statistical Society, 1969, 31, 486-498.

GREEN, D. M., \& SWETs, J. A. Signal detection theory and psychophysics. New York: Wiley, 1966.

Helson, H. The tau-effect: An example of psychological relativity. Science, 1930, 71, 536-537.

Helson, H., \& King, S. M. The tau effect: An example of psychological relativity. Joumal of Experimental Psychology, 1931, 14, 202-217.

KinchlA, R. A. Detecting target elements in multielement arrays: A confusability model. Perception \& Psychophysics, 1974, 15, 149-158.

Kinchla, R. A., \& Collyer, C. E. Detecting a target letter in briefly presented arrays: A confidence rating analysis in terms of a weighted additive effects model. Perception \& Psychophysics, 1974, 16, 117-122.

Price-Whliams, D. R. The kappa-effect. Nature, 1954, 173, 363-364.

\section{NOTE}

1. The Collyer (1976) paper and the present paper are based on sections of a doctoral dissertation submitted to the faculty of Princeton University. The author would like to thank the thesis and orals examination committees of the Department of Psychology for their constructive guidance and criticism. The committee members were Professors Ron Kinchla, Tom Trabasso, Jerry Rudy, Byron Campbell, and Marc Bornstein.

(Received for publication March 29, 1976; revision accepted February 15, 1977.) 